\title{
Initial report of the osteogenesis imperfecta adult natural history initiative
}

\author{
Laura L. Tosi ${ }^{*}$, Matthew E. Oetgen ${ }^{1}$, Marianne K. Floor ${ }^{1}$, Mary Beth Huber ${ }^{2}$, Ann M. Kennelly ${ }^{1}$, Robert J. McCarter ${ }^{1}$, \\ Melanie F. Rak ${ }^{3}$, Barbara J. Simmonds ${ }^{2}$, Melissa D. Simpson ${ }^{4}$, Carole A. Tucker ${ }^{5}$ and Fergus E. McKiernan ${ }^{6}$
}

\begin{abstract}
Background: A better understanding of the natural history of osteogenesis imperfecta (OI) in adulthood should improve health care for patients with this rare condition.

Methods: The Osteogenesis Imperfecta Foundation established the Adult Natural History Initiative (ANHI) in 2010 to give voice to the health concerns of the adult Ol community and to begin to address existing knowledge gaps for this condition. Using a web-based platform, 959 adults with self-reported Ol, representing a wide range of selfreported disease severity, reported symptoms and health conditions, estimated the impact of these concerns on present and future health-related quality of life (QoL) and completed a Patient-Reported Outcomes Measurement Information System (PROMIS ${ }^{\oplus}$ ) survey of health issues.

Results: Adults with OI report lower general physical health status $(p<.0001)$, exhibit a higher prevalence of auditory (58\% of sample versus $2-16 \%$ of normalized population) and musculoskeletal (64 \% of sample versus 1-3\% of normalized population) concerns than the general population, but report generally similar mental health status. Musculoskeletal, auditory, pulmonary, endocrine, and gastrointestinal issues are particular future healthrelated QoL concerns for these adults. Numerous other statistically significant differences exist among adults with $\mathrm{Ol}$ as well as between adults with $\mathrm{Ol}$ and the referent PROMIS ${ }^{\oplus}$ population, but the clinical significance of these differences is uncertain.

Conclusions: Adults with OI report lower general health status but are otherwise more similar to the general population than might have been expected. While reassuring, further analysis of the extensive Ol-ANHI databank should help identify areas of unique clinical concern and for future research. The OI-ANHI survey experience supports an internet-based strategy for successful patient-centered outcomes research in rare disease populations.
\end{abstract}

Keywords: Osteogenesis imperfecta, PROMIS ${ }^{\oplus}$, Patient centered outcomes, Childhood onset conditions, Adult

"Progress can be made when patients and researchers collaborate towards a common goal [1]."

\section{Background}

Osteogenesis imperfecta $(\mathrm{OI})$ is a heterogeneous group of inherited connective tissue diseases defined clinically by excessive skeletal fragility and recurrent fracture [2]. With an estimated prevalence of 8 cases per 100,000 persons, perhaps 30,000 live with OI in the United States [2]. The Sillence classification for OI, first described in

\footnotetext{
*Correspondence: Ltosi@cnmc.org

'Division of Orthopedics and Sports Medicine, Children's National Health System, 111 Michigan Ave NW, Washington, DC 20010, USA

Full list of author information is available at the end of the article
}

1979, includes four types (I-mild, II-neonatal lethal, III-severe, progressively deforming, and IV moderately severe), and is almost exclusively based on physician perception of disease severity [3].

Most OI cases are caused by mutations in the type I collagen genes and are the result of autosomal dominant inheritance. During the past several years, the spectrum of OI has expanded to include rare, recessively inherited forms resulting from abnormalities in the post-translational modification of type I collagen, as well as abnormalities in regulatory proteins involved in bone matrix homeostasis, including those of the Wny-signaling pathway [4, 5]. These non-classical forms of OI can range from moderate severity to neonatal lethality in presentation. 
While the cardinal clinical manifestation of OI is fracture [2], deficiencies in collagen-rich tissues other than bone can lead to abnormal dentition [6], joint laxity [7, 8], hearing loss [9], ocular disease [10], pulmonary [2], and vascular and valvular heart disease [11]. Disease severity ranges from perinatal death to minimally symptomatic forms that escape detection into adulthood [2]. Heightened diagnostic awareness and improved treatments, particularly in severe forms, has increased the number of adults living with OI [12]. While adolescents and young adults with OI may be medically sophisticated, their transition to a new adult health care provider and system of care can be daunting. Adult health care providers and systems have a paucity of medical evidence to guide them and likely little first-hand experience with this condition [13]. Similarly, physically accessible, high quality, informed health care may not be readily available to many [14] and some adults with OI feel that the strong association of OI with "brittle bones" diverts attention away from other important adult health care concerns.

Limited research exists regarding the health issues of adults with OI $[7,8]$. Although adults with OI report a high level of life satisfaction, any conclusions drawn from available reports are constrained by the small number of subjects, the focus on milder forms of OI, and the absence of disease specific quality of life (QoL) measures. Thus, adults with OI, their families and their health care providers cannot formulate expectations of their future health-related QoL, nor anticipate their long-term health care needs. Moreover, despite the potential for mortality and significant morbidity suffered by OI patients, all measures of OI outcomes have been developed by experts without input from patients and rely on clinician-based assessments rather than actual patient reports.

Directly engaging the OI community in patient-centered outcomes (PCO) research could mitigate some of these challenges $[1,15]$. To address concerns raised by the adult OI community and to begin closing the knowledge gap, the Osteogenesis Imperfecta Foundation (OIF) assembled a committee composed of adults with OI (including a practicing physician), orthopedic and medical specialists with extensive experience in the care of persons with OI, OIF representatives, research scientists, and statisticians and created the Osteogenesis Imperfecta Adult Natural History Initiative (OI-ANHI) in 2010. The OI-ANHI tested the feasibility of using web-based, PCO methods for rare disease research by creating an on-line health survey to (1) define health care concerns and perceptions of adults with OI; (2) identify health-care related issues that may have been previously missed or under-valued by adults with OI and their medical providers; and (3) compare QoL responses by adults with OI with those of benchmark populations without OI. This paper reports the initial results of the OI-ANHI survey.

\section{Methods}

Based on adult focus group meetings conducted by OIANHI committee members at the 2010 bi-annual OIF meeting for persons with $\mathrm{OI}$ and their families, a webbased survey was designed and implemented to capture the health status, concerns, needs, and priorities of adults with OI. The complete OI Adult Natural History Initiative survey is available as Additional file 1. The survey was presented in the following sequence: (1) collection of general anthropomorphic, demographic, and medical information; (2) an organ-system-based, medical "review of systems" (ROS) followed by a 5-point Likert ranking to assess the impact of each organ-system on current and expected future QoL; (3) multiple instruments from the PatientReported Outcomes Measurement Information System (PROMIS ${ }^{\oplus}$ ) to evaluate the level of health and functioning for core physical, mental, and social health constructs that are normed to the US general population [16].

Anthropometric data included height and weight. Demographics included year of birth, gender, race/ethnicity, education level, and current country of residence. Medical information addressed medical diagnoses, testing and interventions, and various lifestyle factors. Data collected include OI Sillence Classification, additional medical diagnoses, primary means of mobility, list of health care providers, surgical health interventions, and level of physical activity among other variables.

We queried severity of OI in three different ways: first, respondents provided their understanding of their Sillence type; secondly, they reported whether they perceived their disease to be mild, moderate or severe; height provided an objective measure that has been shown to correlate with disease severity [17].

The second portion of the survey was a medical review of systems (ROS), developed as a collaborative effort by the group of authors, which included physicians, persons with OI, and their advocates, yielding a comprehensive, textbook-quality medical ROS and potentially identifying previously underemphasized or unidentified health concerns. (Additional file 1) We assessed respondents' physical health within fifteen organ systems: neurologic, urinary, musculoskeletal, auditory, cardiovascular, skin, vision, dental, pulmonary, gastrointestinal, endocrine, hematologic, oral, obstetric/gynecologic (females only), and male sexual function (males only). We then used a tri-part format to assess the impact of each system on the respondent's QoL. The first question asked whether or not the respondent had any of the concerns listed specific to the system (e.g. for the skin, rashes and pressure sores; for the pulmonary system, shortness of breath, pneumonia, etc.). The second and third questions for each organ system queried respondents via a 5point, Likert rating scale as to how much issues within the relevant organ system currently impacted their QoL 
and how much they thought problems with each system would impact their quality of life in the future.

The third and final portion of the survey used selected $\mathrm{PROMIS}^{\odot}$ item banks to evaluate general physical health, pain, fatigue, physical function, sleep, depression, anxiety, sexual satisfaction, and social function. Point values for $\mathrm{PROMIS}^{\circ}$ construct responses were calibrated to T-scores generated from a representative U.S. population and reported on a 100-unit scale where the mean is set at 50 units, with 10 units representing one standard deviation (SD). This data transformation follows the guidelines set forth in PROMIS methodology [18]. Means and $95 \%$ confidence intervals for the OI population were calculated based on individual T-scores for a given concept. The minimum important difference (MID) in $\mathrm{PROMIS}^{\oplus}$ responses thought to be clinically relevant has been estimated as $0.3-0.5$ SD for fatigue, anxiety and depression; 0.4-0.6 SD for pain; and 0.2 for physical function $[19,20]$.

Participants accessed the survey through a web-based portal and were recruited with two OIF postal mailings, a notice posted on the OIF web-site, the OIF newsletter Breakthrough, spoken and written notification at national and regional clinical and scientific meetings, social networks (Facebook, Twitter, OI forums and chat-rooms), and word of mouth. Children's National Health System's Institutional Review Board exempted the survey from review, as it was anonymous. Prior to participation, respondents read the study's purpose, gained study contact information, learned of the right to withdraw, and then chose either to participate or not participate. The survey instrument was available from $10 / 01 / 2011$ to $12 / 31 / 2011$ and designed so that respondents could complete the survey progressively. Those without computer access or facility were offered a paper version, and a data entry specialist masked to subject identity uploaded completed results. Paper surveys were accepted until midnight 01/02/2012. The survey required at least one hour, but more than likely, two hours for completion. Only respondents who answered at least one question in the final PROMIS ${ }^{\circ}$ item set (Sleep Disturbance) were considered to have completed the entire OI-ANHI survey.

Body Mass Index (BMI) was calculated as $\mathrm{kg} / \mathrm{m}^{2}$ and classified according to the World Health Organization (WHO) weight classification criteria [21]. Height Z-scores were calculated from the National Health and Nutrition Examination Survey (NHANES III) dataset [22]. Height $\mathrm{Z}$-scores indicate the number of SD a reported height varies from a US adult, gender matched mean. To compare the PROMIS scores of the generalized population with those of our sample, we segregated PROMIS ${ }^{\circ}$ T-scores by both OI self-reported severity grouping (mild, moderate, severe) and by quartile of height Z-score. For continuous variables, a $\mathrm{t}$-test was used to calculate the $p$-value; for dichotomous variables, a chi-square test was used. For variables reported on a Likert scale, a generalized linear model was utilized (SAS proc glm). Generalized linear models were also used to compare differences among OI groups. All analyses were performed using SAS version 9.2 (SAS institute, Cary NC).

\section{Results}

Of 1,183 individuals who initiated the survey, 959 (82\%) successfully completed it, constituting the study sample. Demographically, respondents were female $(71 \%)$, middle aged (mean $45.1 \pm 15.0$ years, range 18-89 years), Caucasian (91 \%) and lived in North America (89\%). Figure 1 is a graphical depiction of age distribution. Age did not vary on the basis of self-reported severity grouping (Fig. 2). Highest educational achievement exceeded secondary school for $82 \%$. In regard to anthropometric data, average height was $57.3 \pm 9.2$ inches, weight $135.9 \pm 44.6$ pounds, and BMI $29.4 \pm 9.3$.

In response to the OI-specific medical questions, the majority walked unaided (61 \%), but $16 \%$ required a cane, crutches or walker, and nearly one-quarter reported regular wheelchair use. The majority had received their OI diagnosis from a doctor (59\%). Respondents considered their OI to be mild (56\%), moderate (35\%) or severe $(9 \%)$ and reported Sillence classifications of Type I (39 \%), Type III (13\%), and Type IV (12\%). Fewer than $5 \%$ described other Sillence types and $31 \%$ reported their type to be unknown. Table 1 shows the relationship between self-reported disease severity and self-reported Sillence type. As self-reported disease severity worsened, height Z-score decreased ( $\beta=-0.13, p<0.0001$, Fig. 3 ).

A large proportion of participants $(65 \%)$ consumed vitamins or dietary supplements, followed by pain $(40 \%)$ and blood pressure medications (32\%). Medical providers reported to be actively involved in respondents' medical care included a "general medical doctor" (92\%), orthopedist (49\%), gynecologist (40\% of women), and endocrinologist $(21 \%)$. Cardiologists, dermatologists and gastroenterologists were involved in $10 \%$ of

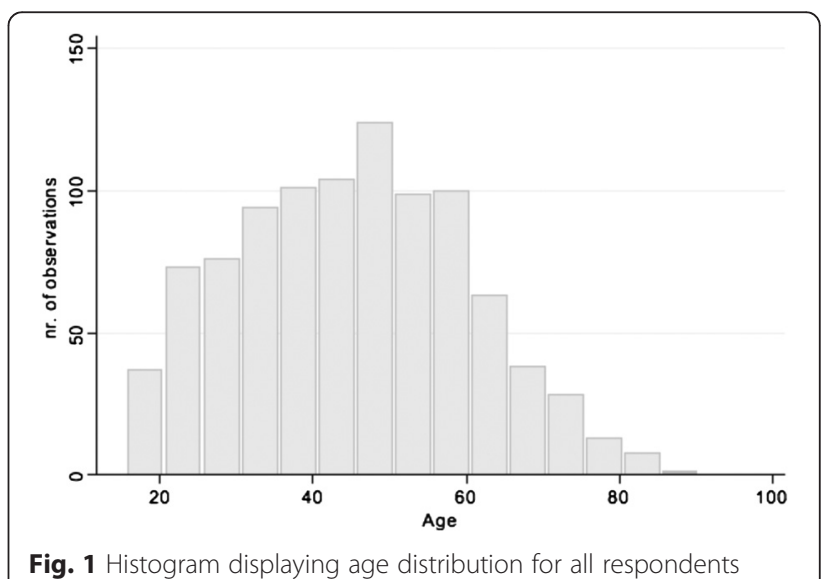




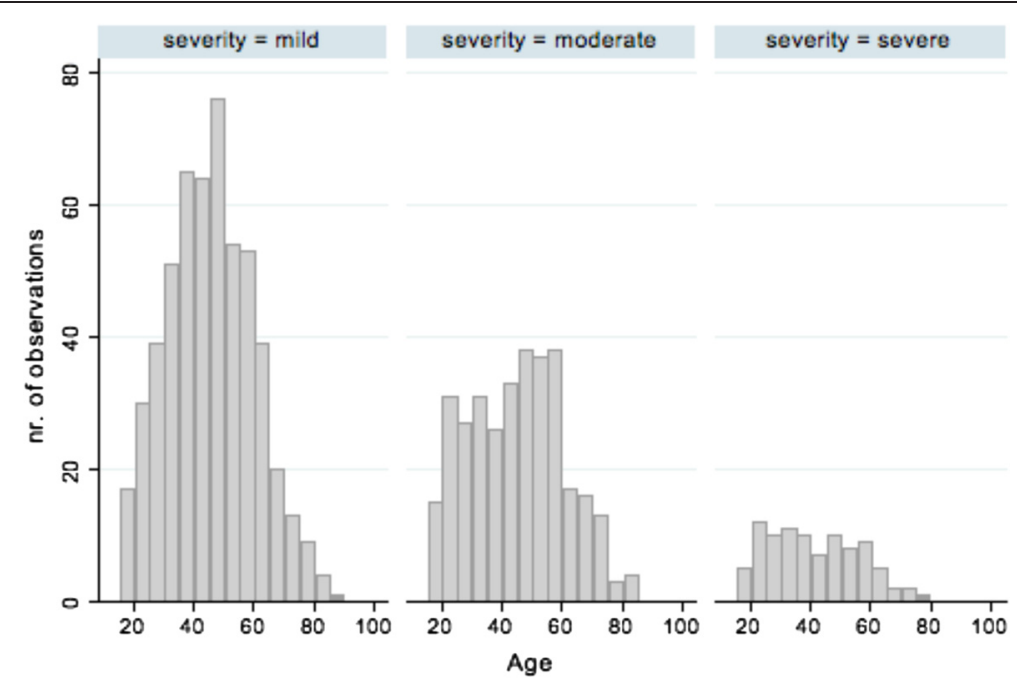

Fig. 2 Histogram depicting age in five-year groups by self-reported severity grouping. Three categories of severity are reported: mild, moderate, and severe

respondents' care, but few other medical specialists had ever been engaged. Other providers were dentists $(68 \%)$ and ophthalmologists (30\%). Both overall and when separated by self-reported severity grouping, respondents saw a median number of 4 different care providers. The overall range was 0 to 14 (Fig. 4). Number of health care providers seen did not vary on the basis of self-reported OI severity grouping. Notably, just over half of participants reported no health interventions (52\%), while $35 \%$ had received rodding surgery. Other than the two aforementioned responses, respondents indicated a scant number of other health interventions. Many of the respondents had received medical testing in the prior year with $86 \%$ reporting a blood pressure reading, $71 \%$ a vision exam, $66 \%$ a blood test for cholesterol, and $34 \%$ a DXA scan. Respondents reported very few cancer

Table 1 Relationship between self-reported Sillence type and self-reported disease severity in adults with $\mathrm{Ol}$

\begin{tabular}{lcccc}
\hline & & \multicolumn{3}{l}{ Self-reported disease severity } \\
\cline { 3 - 5 } Ol type & $\mathrm{n}(\%)$ & \multicolumn{1}{c}{ Mild } & Moderate & Severe \\
\hline I & $369(39)$ & $308(83)$ & $59(16)$ & $2(<1)$ \\
II & $26(3)$ & $12(46)$ & $8(31)$ & $6(23)$ \\
III & $125(13)$ & $7(1)$ & $67(20)$ & $51(57)$ \\
IV & $110(12)$ & $45(41)$ & $55(50)$ & $10(9)$ \\
V & $14(1)$ & $2(14)$ & $11(79)$ & $1(7)$ \\
VI & $2(<1)$ & - & $1(50)$ & $1(50)$ \\
Bruck's syndrome & $3(<1)$ & - & $3(100)$ & - \\
Unknown & $295(31)$ & $154(52)$ & $123(42)$ & $18(6)$ \\
Total & $944(100)$ & $528(56)$ & $327(35)$ & $89(9)$ \\
\hline
\end{tabular}

Missing $n=15$ diagnoses, with the majority reporting none (90 \%) followed by skin cancer (4\%).

Respondents acknowledged few general health concerns, with "none of these" (52\%) as the most endorsed response followed by changes in sleep patterns $(27 \%)$ and mood swings (20\%). Almost half of respondents (49 $\%)$ engaged in walking as a regular physical activity although approximately one-third (34\%) reported that they do not exercise regularly. There was a significant relationship between engagement in physical activity and severity grouping (Fig. 5). The most popular exercise

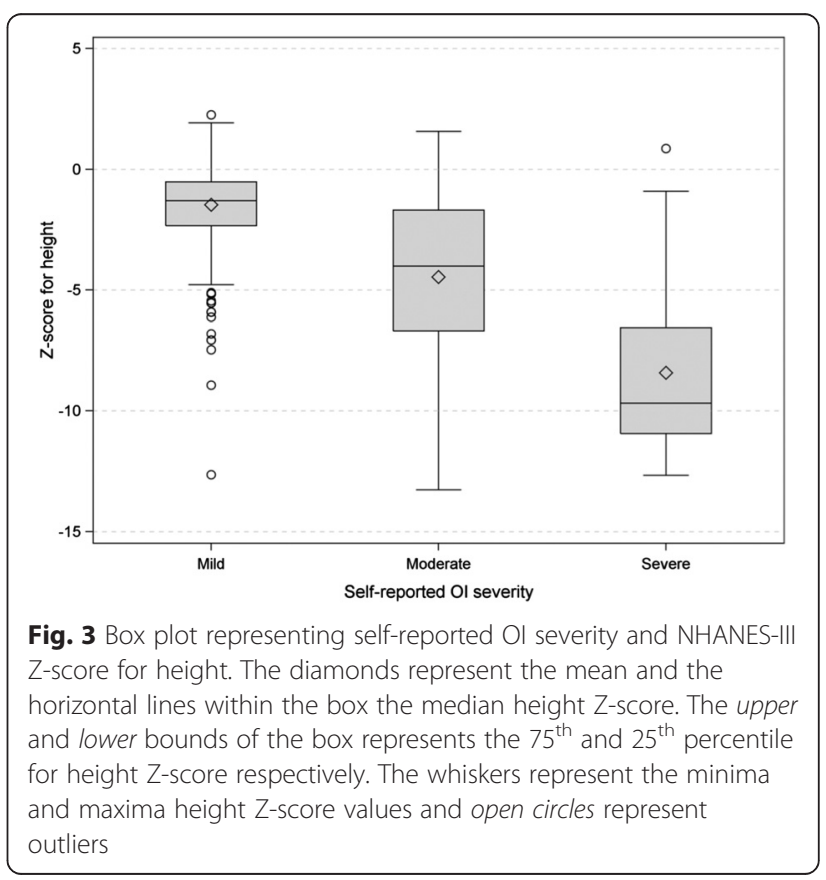




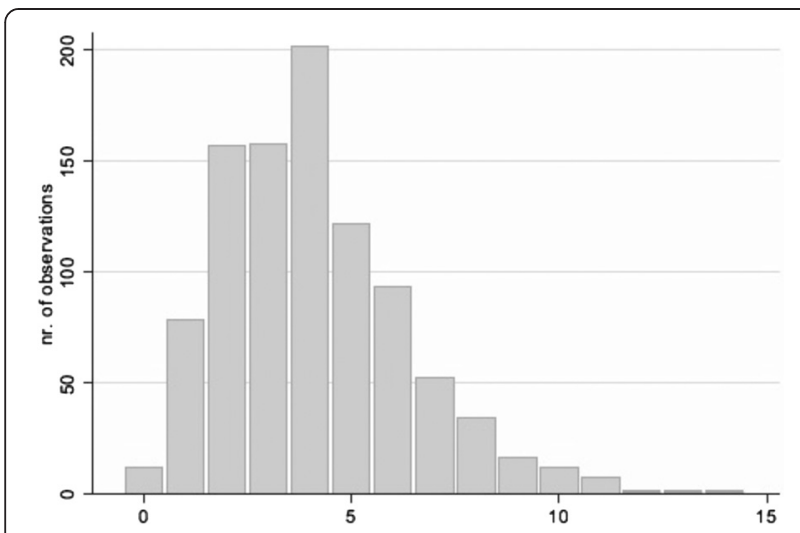

Fig. 4 Histogram displaying number of healthcare providers seen for all respondents

venue was the home (51\%), and respondents oftentimes developed their own exercise programs (40\%).

Only $2 \%$ of respondents reported having no medical provider and $4 \%$ considered urgent care or emergency room providers to be their primary source of health care. Ninety-one percent of respondents had health care insurance. Many adults with OI turn to their medical providers for health-related information (53\%); however, more rely on the Internet (63\%) and the OIF (71\%) for that information. Indeed, only $32 \%$ indicated "quite a bit" or "very much" confidence in their primary care provider's medical management of their OI. Approximately one-third of respondents reported no regular dental care.

Table 2 summarizes prevalence for various self-reported conditions and symptoms, segregated by self-reported disease severity, compared to similar results reported in the NHIS 2012 [23] and other benchmark datasets [24-38].
Musculoskeletal problems (particularly fractures), hearing loss, heartburn, and astigmatism differ most between OIaffected adults and the general population. Reports of dry skin, numbness, sleep apnea, hemorrhoids and shortness of breath appear to be more prevalent in adults with OI. Only fracture, hearing loss and to some extent heartburn and constipation relate to OI severity. In regard to obstetric/gynecological health, an unexpected finding was that one-third of adult women with severe OI reported a prior pregnancy, with only $6 \%$ reporting miscarriage and none reporting stillbirths. Among the women in our sample 55 years of age and younger, those reporting severe OI experienced significantly less $(p<.05)$ catamenial bleeding than those with mild or moderate OI.

The organ systems most often reported to affect current QoL are urinary tract (97\%), musculoskeletal (95\%), vision (82\%), auditory (75\%), dental (74 \%), skin (68\%), gastrointestinal (65\%), and neurological (64\%). Using a Likert scale, Fig. 6 shows the impact of specific organ system concerns on current and anticipated future QoL for all respondents (Fig. 6 left) and for those already experiencing problems within that organ system (Fig. 6 right). Disproportionate concern was reported for future pulmonary, cardiologic and vision issues by all respondents, and for endocrine, pulmonary and cardiovascular issues in those respondents already experiencing issues in these domains.

Figure 7 shows the mean PROMIS ${ }^{\circ} \mathrm{T}^{-}$-scores of selected items by self-reported OI severity compared with the PROMIS ${ }^{\circ}$ population norm. General physical health status was significantly lower than that of the general population and the magnitude of difference (0.4-1.0 SD) exceeded the MID established for several PROMIS ${ }^{\circ}$ domains $[19,20]$. General physical health among the OI cohort was lower in those reporting greatest disease

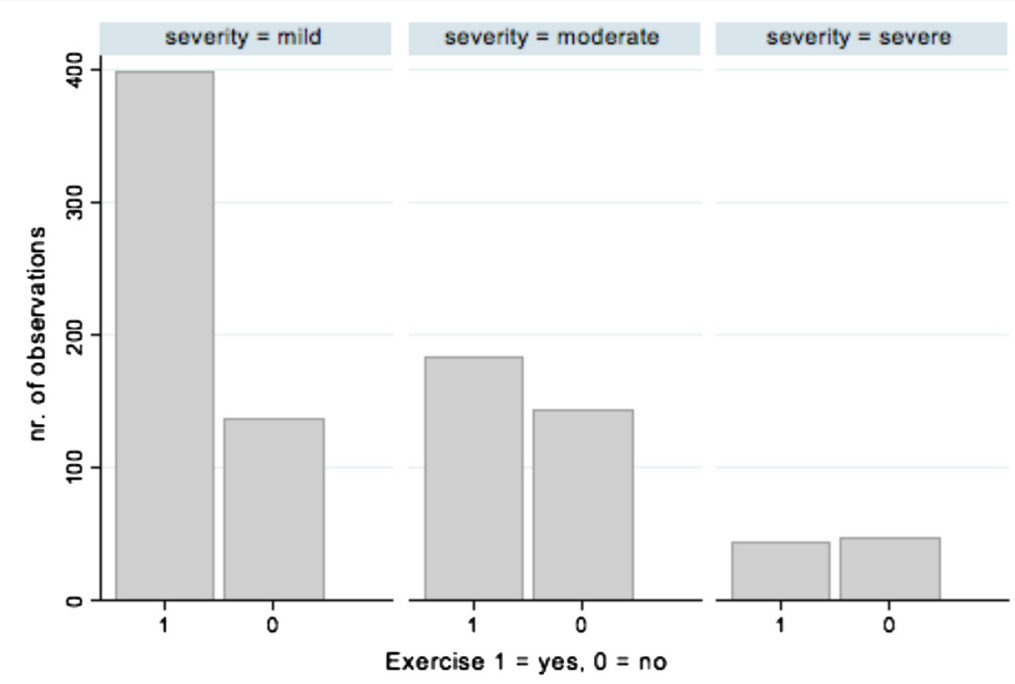

Fig. 5 Histogram depicting engagement in or disengagement from exercise by self-reported severity grouping. A response of "1" correlates with "yes" for exercise while "0" represents a response of "no." Three categories of severity are reported: mild, moderate, and severe 
Table 2 Prevalence of self-reported conditions and symptoms by adults with Ol shown by self-reported disease severity compared with prevalence reported by both genders in NHIS 2012 or, where specified, in other adult benchmark databases

\begin{tabular}{|c|c|c|c|c|c|}
\hline \multirow[t]{3}{*}{ Condition } & \multirow{3}{*}{$\begin{array}{l}\text { Mild OI } \\
(n=535)\end{array}$} & \multirow{3}{*}{$\begin{array}{l}\text { Moderate OI } \\
(n=329)\end{array}$} & \multicolumn{3}{|c|}{ Prevalence of reported conditions and symptoms (\%) } \\
\hline & & & \multirow{2}{*}{$\begin{array}{l}\text { Severe OI } \\
(n=92)\end{array}$} & \multirow{2}{*}{$\begin{array}{l}\text { All } \\
(n=959)\end{array}$} & \multirow[t]{2}{*}{ NHIS 2012 [23] } \\
\hline & & & & & \\
\hline Astigmatism & 33 & 37 & 30 & 34 & $3.6[24]$ \\
\hline Blood vessel problem (rupture, aneurysm) & 5 & 6 & 10 & 6 & $1-12[25]$ \\
\hline Bruising & 47 & 41 & 15 & 42 & $18(12-55)$ \\
\hline Cancer & 11 & 9 & 4 & 10 & $5-8[26]$ \\
\hline Cataracts & 8 & 9 & 11 & 8 & $8.6[25]$ \\
\hline Constipation & 23 & 29 & 34 & 26 & $12-19[27,28]$ \\
\hline \multirow[t]{2}{*}{ Coronary artery disease } & 6 & 11 & 8 & 4,7 & $5-17(<65 y r)$ \\
\hline & 20 & 12 & 0 & 10,10 & 30 (>65 yr) [25] \\
\hline Heart attack & 6 & 9 & 7 & 7 & $2.9(>20 \mathrm{yr})[25]$ \\
\hline Cough & 11 & 19 & 30 & 16 & 9-33 [29] \\
\hline Diabetes mellitus & 11 & 11 & 8 & 11 & $8-10[23]$ \\
\hline \multirow[t]{2}{*}{ Dry skin } & 35 & 30 & 28 & 32 & $14-19(<65 y r)$ \\
\hline & 49 & 62 & 0 & 52 & 56 (>65 yr) [30] \\
\hline Fractures & 59 & 72 & 66 & 64 & $1-3[31]$ \\
\hline Fragile teeth & 29 & 46 & 57 & 38 & 10-19 [32] \\
\hline Glaucoma & 8 & 7 & 2 & 7 & $2[24]$ \\
\hline Hearing loss & 59 & 57 & 58 & 58 & $2-16$ [23] \\
\hline Heart valve problem & 6 & 9 & 4 & 7 & $2-4[25]$ \\
\hline Heartburn & 30 & 40 & 30 & 33 & $3-7[33]$ \\
\hline Hemorrhoids & 17 & 18 & 11 & 17 & $4[28]$ \\
\hline High cholesterol & 29 & 27 & 12 & 26 & $8-27[23,25]$ \\
\hline Hypertension & 32 & 42 & 34 & 35 & $21-36[34]$ \\
\hline Kidney/bladder stones & 8 & 14 & 4 & 9 & $8.8[35]$ \\
\hline Near sightedness & 50 & 49 & 39 & 49 & $33-40[24]$ \\
\hline Numbness & 18 & 22 & 25 & 20 & $0.1-8[36]$ \\
\hline Obesity (BMI > $30 \mathrm{~kg} / \mathrm{m}^{2}$ ) & 29 & 42 & 50 & 35 & $28-35[23]$ \\
\hline \multirow[t]{2}{*}{ Shortness of breath } & 20 & 31 & 47 & 26 & $10-18(<65 \mathrm{yr})$ \\
\hline & 28 & 31 & 60 & 31 & $30+(>65 y r)[37]$ \\
\hline Sleep apnea & 9 & 17 & 32 & 14 & $<6[38]$ \\
\hline Stroke & 3 & 6 & 8 & 4 & $3[25]$ \\
\hline Wheezing/asthma & 12 & 14 & 27 & 14 & $8-13$ [37] \\
\hline
\end{tabular}

NHIS 2012: "Any hearing trouble without use of hearing aid or listening device"

severity but the magnitude of this difference was small. Results also revealed small but statistically significant differences between adults with OI and the normative population for a number of the PROMIS ${ }^{\bullet}$ scales; adults with OI reported greater levels of anxiety $(p<.0001)$ and depression $(p<.0001)$, as well as lower levels of general mental health $(p<.0002)$. However, the differences in means between adults with OI and the normative population for these three scales did not qualify for clinical relevance according to the established MIDs $[19,20]$.
Segregation of PROMIS ${ }^{\circ}$ T-scores by quartile of height Z-score (Fig. 8) demonstrates results similar to Fig. 7 and shows that decreased height does not consistently correlate with less desirable $\mathrm{PROMIS}^{\circ}$ scores within our adult OI sample for certain scales, including access to help and satisfaction with social roles.

Remarkably, adults with OI report lower pain intensity than the general PROMIS ${ }^{\bullet}$ population. While adults with OI indicate that pain interferes with their functional state significantly more than it does for the general 


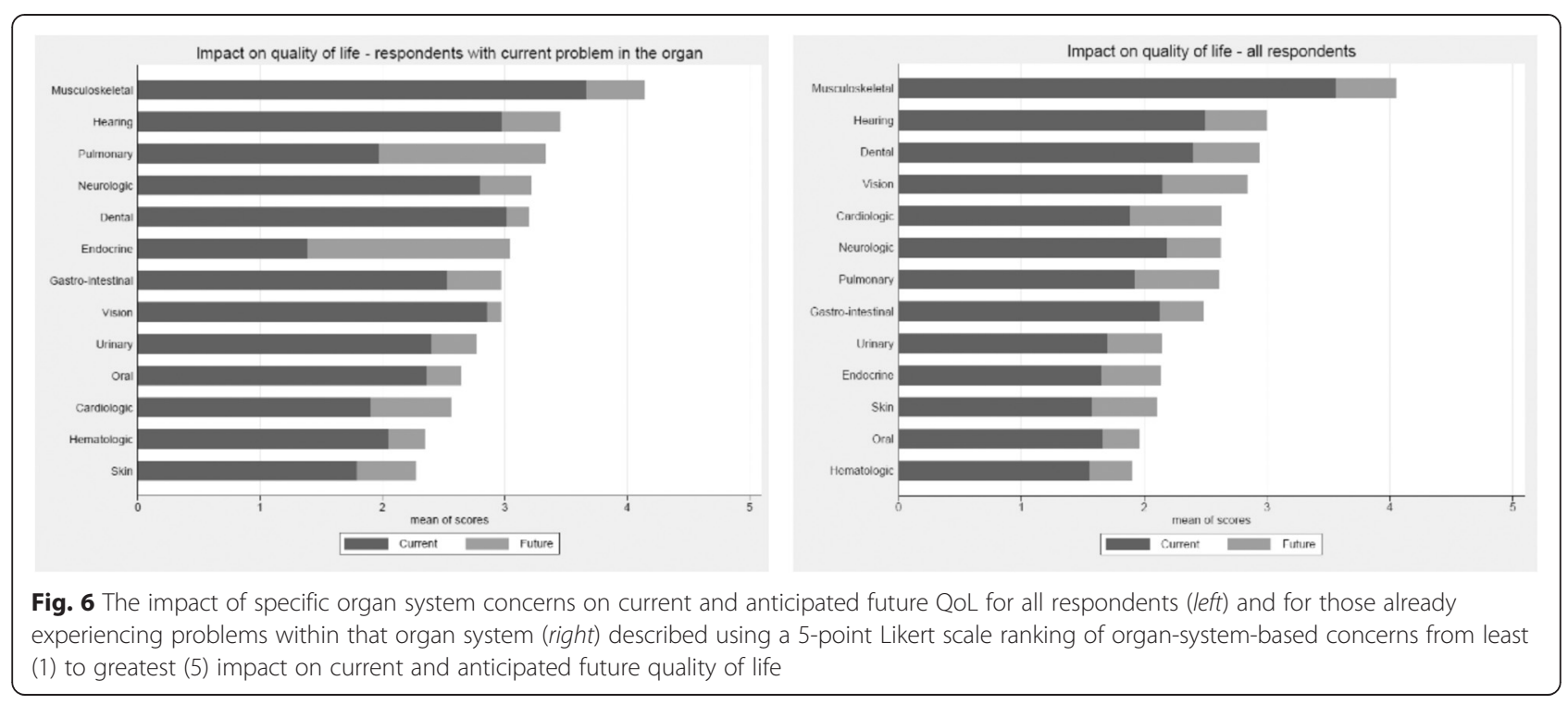

PROMIS ${ }^{\oplus}$ population, this difference appears to be less than the MID and results did not differ by self-reported disease severity.

Only those adults with severe OI appeared to report notably (-0.56 SD) lower satisfaction with sexual activity. Statistically significant differences in T-scores for responses to "sleep disturbance", "satisfaction with social roles" "fatigue", "access to help", and "ability to participate in social activities" were found between the OI cohort and the general PROMIS $^{\oplus}$ population but these differences appear to be less than a MID.

\section{Discussion}

Delineating and meeting the health needs of adult survivors of childhood-onset conditions is a growing qualityof-care issue. Based on estimates of those living with OI in the US ranging from 25,000 [39] to 50,000 [40], approximately $3 \%$ of the total US OI population completed the OI ANHI survey. This number is especially impressive given the fact that adults with $\mathrm{OI}$ are a rare, geographically dispersed population and that we recruited respondents in an incentive-free manner. In doing so, we have demonstrated how persons with rare (and not so rare) conditions can collectively address some of their important health related concerns as a virtual community.

\section{Demographics and medical/lifestyle information}

BMI exceeded the WHO diagnostic threshold for obesity $\left(30 \mathrm{~kg} / \mathrm{m}^{2}\right)$ in $36 \%$ of respondents and an additional 31 $\%$ were overweight. While this prevalence is similar to the general adult US population, it is unclear that this finding conveys the same health risk for an individual whose height is 5 or more SDs below average. Hypertension has been reported to be more prevalent in adults with OI but this was not the case in our population.
Unexpectedly, adults with self-reported severe OI reported substantially less catamenial bleeding than those reporting mild or moderate disease. Heightened vigilance for cutaneous trauma or the protective effect of a wheelchair might explain the former but could not account for the latter. While vague, the large number of respondents reporting numbness suggests the need for further inquiry as the incidence of basilar invagination, a potential explanation for numbness, is much higher in persons with OI than the general population.

Neither age nor number of health care providers seen varied by self-reported OI severity grouping. The finding regarding age was surprising as we anticipated that those individuals with more severe forms of OI would be younger because of the higher number of medical and musculoskeletal problems that can accompany more severe disease. While those with the milder types of OI, such as types I and IV, can anticipate a normal lifespan, prior research has found a reduced lifespan for those with type III OI [12, 41].

The findings that age does not vary on the basis of the subjective self-reported grouping and that height is an inconsistent predictor of quality of life suggest the same outcome: there are important aspects of OI severity and functioning not captured by some of the markers relied upon in the past, including age and height. These inconsistencies underscore the need to validate the PRO$\mathrm{MIS}^{\odot}$ instruments for use in OI and to develop diseasespecific QoL measures in order to better assess functioning in adults with OI. Satisfaction with sexual activity was the only construct that revealed statistically significant differences between groups.

We determined significant differences in exercise engagement based on perceived disease severity. This finding deserves further inquiry. Given that this was a 


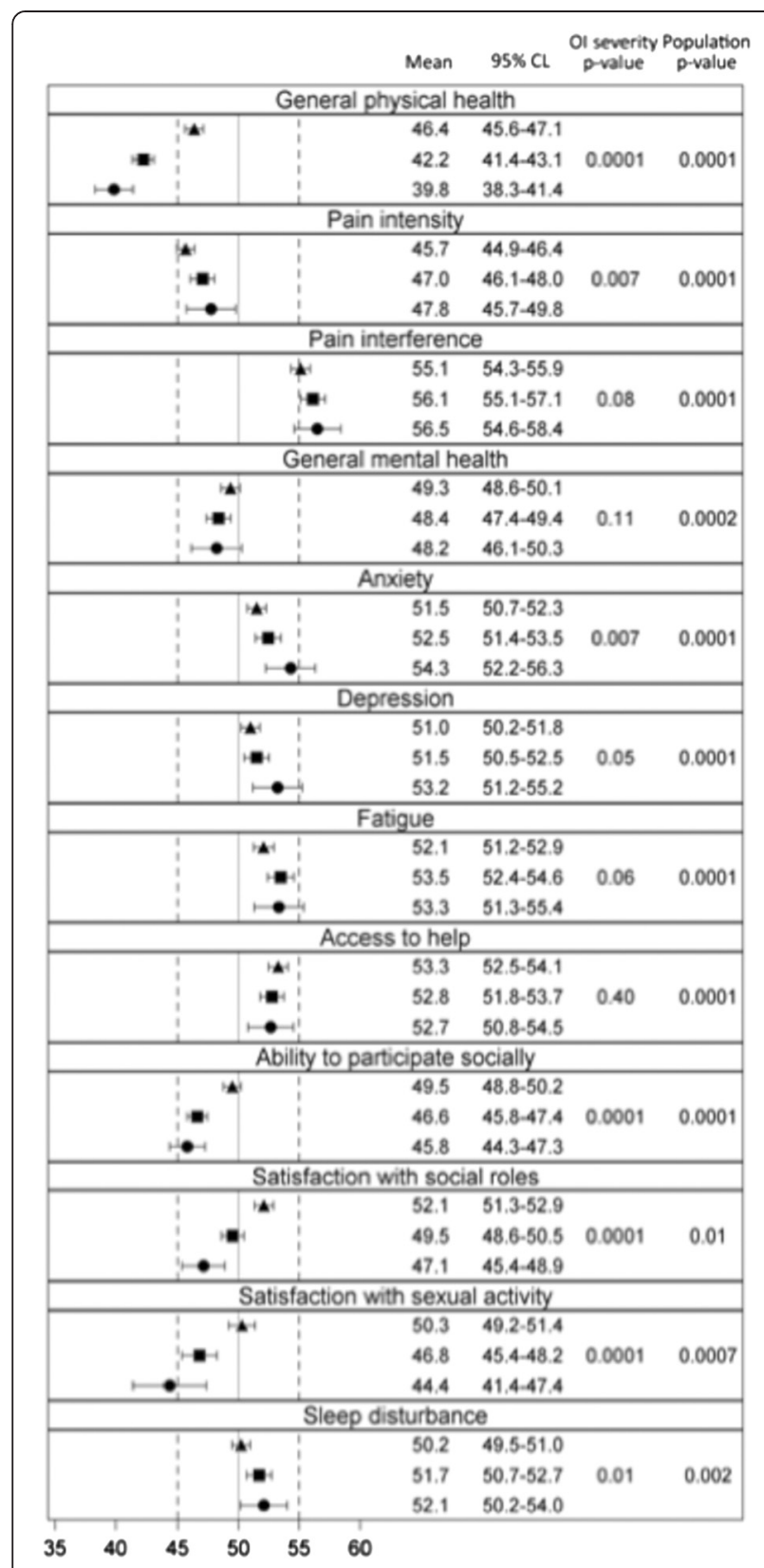

Fig. 7 Mean PROMIS ${ }^{\oplus}$ T-scores with 95\% confidence limits by selfreported OI severity. Symbols represent self-reported OI severity ( $\boldsymbol{\Lambda}=$ mild, $\boldsymbol{\|}=$ moderate, $\bullet=$ severe). The mean T-scores of the PROMIS referent population are indicated by the solid vertical

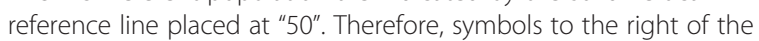
reference line are higher than the PROMIS ${ }^{\oplus}$ population mean (i.e. more of that construct than the PROMIS ${ }^{\star}$ population) and symbols to the left of the reference line are lower than the PROMIS ${ }^{\circledR}$ population mean (i.e. less of that construct than the PROMIS ${ }^{\circledR}$ population). $p$-values are for the comparisons of T-scores within disease severity strata amongst the entire OI cohort (left column, "Ol severity") and between the entire Ol cohort and the general PROMIS ${ }^{\oplus}$ population (right column, "Population") self-reported disease severity rating rather than Sillence classification, do adults who report more severe OI indeed abstain from physical activity because of the severity of their condition or are there individuals with more mild forms of OI reporting greater OI severity because their condition has potentially also worsened from exercise abstinence? Previous research among adults with OI determined a correlation between decreased physical function and ability to participate in the physical actions of climbing stairs and going for walks [8]. Similarly, a study among pediatric patients with OI found that walking ability suffered with greater OI disease severity [42]. Such research suggests that physical activity participation is not only a challenge in OI, but also becomes less likely with augmented OI severity.

However, physical activity, especially physiotherapy involving muscle strengthening, is an important aspect of OI management [8] and physical activity has been shown to improve functioning in pediatric populations with mild to moderate OI [43]. Unfortunately there are no studies to guide persons with severe OI. In light of our finding that adults with self-reported, severe OI show greater disengagement from exercise, future studies should examine this association and its determinants. In turn, longitudinal research should examine the potentially protective effect of exercise by all types of OI disease severity.

\section{Organ-specific quality of life concerns}

Adult OI respondents indicate that musculoskeletal and auditory issues have a greater impact on current and anticipated future QoL than other organ system concerns, and express even greater concern about the future impact of endocrine and pulmonary issues on QoL. Respiratory failure is a leading cause of death in severe OI $[12,41]$. The lungs are often greatly affected in OI, with challenges such as pulmonary restrictive disease due to chest wall or pulmonary collagen abnormality, airway obstruction, pulmonary hypertension, and sleep apnea [44]. Care recommendations have been developed by Sandhaus, which include intensive, longitudinal followup of pulmonary function in patients with OI [44]. This is particularly important given the high rates of sedentarism and elevated BMI reported [12, 45]. Therefore, the low rate of reported pulmonary consultation could be an opportunity for improving care and health status.

Up to $50 \%$ of adults with OI have dentogenesis imperfecta [6] yet approximately one-third of our respondents reported no regular dental care, and $74 \%$ expressed current QoL concerns regarding their dental health. An important future research direction should include a more in-depth analysis of dental care in OI to establish whether adults with OI have difficulty gaining access to dental care or whether there is another explanation for this shortfall. 


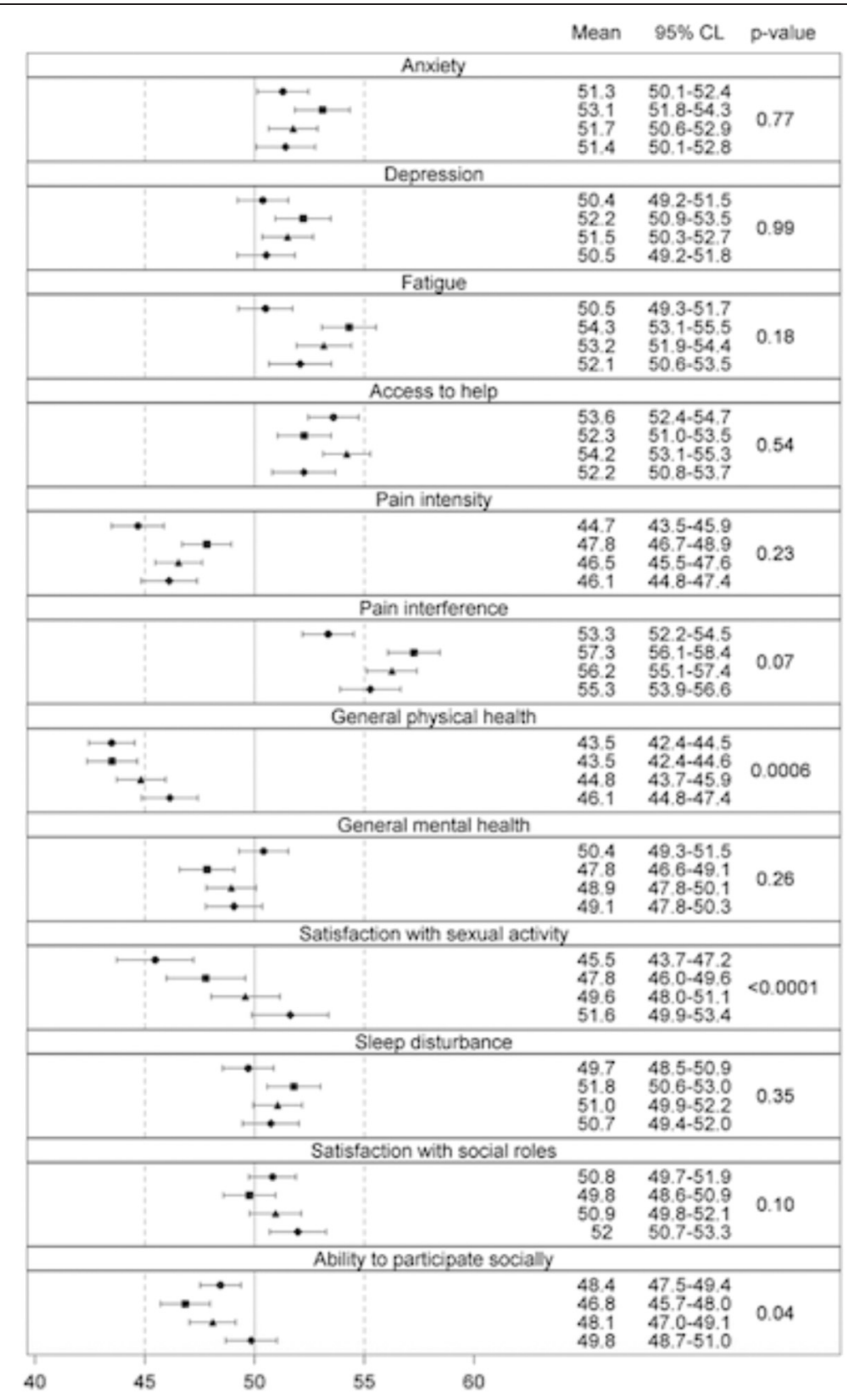

Fig. 8 Mean PROMIS ${ }^{\oplus}$ T-scores with 95\% confidence limits by quartile of NHANES-III Z-score for height. Symbols represent self-reported OI severity $\left(\boldsymbol{\Lambda}=\right.$ mild, $\boldsymbol{\|}=$ moderate, $\bullet=$ severe). The mean T-scores of the PROMIS ${ }^{\bullet}$ referent population are indicated by the solid vertical reference line placed at " $50^{\circ}$. Therefore, symbols to the right of the reference line are higher than the PROMIS ${ }^{\circledR}$ population mean (i.e. more of that construct than the PROMIS ${ }^{\oplus}$ population) and symbols to the left of the reference line are lower than the PROMIS ${ }^{\oplus}$ population mean (i.e. less of that construct than the PROMIS ${ }^{\circledR}$ population). $p$-values are for the comparisons of T-scores within disease severity strata amongst the entire Ol cohort (left column, "Ol severity") and between the entire Ol cohort and the general PROMIS ${ }^{\circledR}$ population (right column, "Population")

Multiple insights into improving research and care were gleaned from the ROS. Results from the QoL portion of the medical ROS revealed that adults with OI have future-related QoL concerns with organ systems identified as troublesome, particularly the musculoskeletal, auditory, and pulmonary subsystems. In addition, respondents expressed future-related, QoL concerns with the endocrine and gastrointestinal organ systems, subsystems that have not been heavily studied in the existing OI literature. For better incorporation of patients' concerns into care and integration of what doctors know to be important with what matters to patients, exploration of the 
endocrine and gastrointestinal subsystems represents an important future research direction.

\section{Patient-centered/patient-reported outcomes: PROMIS ${ }^{\circledR}$}

As according to $\mathrm{PROMIS}^{\circ}$, adults with OI report lower general physical health status than the general population. General physical health status among respondents reporting the greatest disease severity was lower, but the clinical significance of this finding is uncertain. Numerous statistically significant differences exist among adults with OI of varying severity as well as between adults with OI and the general population, but the clinical significance of these differences is uncertain. Indeed, the PROMIS $^{\circ}$ instruments suggest that, overall, the OI community does not differ greatly from the general population. Moreover, the results of the OI-ANHI survey support previous findings that adults with OI report a high level of life satisfaction in spite of a significant disease burden which, in our collective experience, the authors would characterize as resilience $[7,8,46]$.

We and others have shown significant correlations between disease severity and height Z-score [17], but when PROMIS ${ }^{\circ}$ T-scores are segregated by quartile of height Z-score, our results suggest that these measures may convey slightly different QoL information in this population. Clinicians encounter adults with ostensibly mild OI who consider their condition to be severe when their only objective manifestation of OI is adult-onset deafness. Perhaps "severity" should also be viewed from the patient's perspective and not only by height Z-score or Sillence type. Whether nuances in QoL information conveyed by these different measures are important is unknown but, if so, they could be relevant to the development of a disease-specific QoL instrument for OI. The PROMIS ${ }^{\circ}$ scales are validated and scaled for a healthy, adult population, which represents a challenge in their application to a chronic, rare disease group. Although our results suggest that adults with OI exhibit similarities with the normative population, it is possible that the scales are not capturing some differences within our sample. The validation of PROMIS ${ }^{\circ}$ instruments for persons with OI will be an important future study focus so that researchers can better understand QoL in OI and move towards the application of PROs both in clinical settings and comparative effectiveness research [47].

\section{Limitations}

Most likely, our recruitment and web-based strategies resulted in a self-selection/enrollment bias. We were likely unable to capture the responses of non-Internet users in this study. Nonetheless, our study represents the first effort to query a large sample of adults with OI regarding their disease burden and QoL. Although our study relied heavily on an Internet rather than a paper format, several studies have quantified the reliability of Internet research studies by comparing them to identical studies conducted with non-internet formats and have found them to be equally as reliable [48, 49]. Internet methods have been shown to be as reliable as paper formats when measuring patient-reported health outcomes for those suffering from chronic health conditions [50].

Our results may be limited by the restricted demographic heterogeneity of our sample. Male gender, lower educational achievement status, and non-Caucasian race were all under-represented. Previous research has shown that women generally rely on the Internet for health and medical information more than men [51]. In addition, as of 2015, those with college education are more likely than those who do not have high school diplomas to use the Internet and the minority groups of African American and Hispanic ancestry are less likely to be Internet users than Caucasians [52]. These noted differences in Internet usage may partially explain why our sample proved to be predominantly female, well educated, and Caucasian. However, previous studies examining OI have also noted a higher proportion of female $[7,8,38]$, more highly educated [45], Caucasian [38] respondents, (the majority of whom describe themselves as having type I OI) [38]. Multiple other studies have also reported a middleaged mean [7, 8, 45]. Thus, our study accessed those members of the OI community most often reported in other research studies. One of our future goals is to reach the populations under-represented in this study, namely men, minorities, and those with a lower level of education.

Another limitation of this study was the challenge of defining disease severity. Even before Sillence developed the current OI classification in 1979, efforts to stratify disease severity had been ongoing and continue to be a challenge because of the expanding clinical and genetic heterogeneity found by researchers. At present, 17 different genetic causes of OI have been identified [53] and it is anticipated that more will be elucidated. Therefore, the effort to define disease severity is a continuous struggle in OI research. Furthermore, misclassification of OI type was possible due to the self-report nature of our research. Indeed, $31 \%$ of respondents were unaware of their Sillence type, and twelve respondents characterized their OI as both Type II and mild, which, until recently, was considered lethal.

In this study, however, we attempted to focus less on Sillence classification, as our goal was greater inclusion of the patient's perspective and his or her own assessment of functioning and disease burden. Indeed, it has been documented that physicians and patients sometimes disagree on disease severity in chronic diseases. Lack of concordance between physician and patient ratings of disease severity in rheumatoid arthritis is an apt 
example [54, 55]. Therein, we developed the severity measure for this study in a self-report format. Yet, objective measures of OI severity were not absent from our work. Rather than rely fully on Sillence classification, we included height as an objective marker of disease severity and then examined its relationship with both self-reported disease severity as well as the PROMIS instruments.

Chief among the study's strengths is the very large number of respondents and the $\mathrm{PCO}$ perspective of this inquiry. The success of this survey in reaching a large number of adults with OI has encouraged our research team to explore new strategies to engage the groups that were underrepresented in the survey, namely men, persons of limited educational achievement, and non-Caucasians.

\section{Conclusion}

The OI-ANHI survey engaged an adult OI community to identify issues of specific health-related QoL concern and to focus future research efforts designed to improve quality of care. Our results revealed that while adults with OI exhibit similarities with the normative population, there are important differences, including a higher prevalence of musculoskeletal and auditory problems and specific QoL concerns regarding the musculoskeletal, auditory, pulmonary, and endocrine systems. Our future research will focus on expanding the range of health care topics pursued in OI research and on incorporating patients' QoL concerns into effectiveness evaluations of diagnostic and treatment strategies. Finally, we will strive to develop methodologies that allow us to be more inclusive and engage the broader OI community in our future research studies.

\section{Availability of supporting data}

The data supporting the results of this article are housed in the Osteogenesis Imperfecta Foundation.

\section{Additional file}

Additional file 1: The OI Adult Natural History Initiative survey (OI-ANHI survey). (PDF 291 kb)

\section{Competing interests}

$\mathrm{MBH}$ is an employee of the Osteogenesis Imperfecta Foundation.

\section{Authors' contributions}

LLT, MEO, MBH, AMK, MFR, BJS, CAT, and FEM made substantial contributions to the conception and design of this work and the acquisition, analysis and interpretation of the data. MKF, MDS, and RJM made substantial contributions to the analysis and interpretation of data. All authors were involved in drafting the manuscript as well as revising it critically for important intellectual content and agree to be accountable for all aspects of the work in ensuring that questions related to the accuracy or integrity of any part of the work are appropriately investigated and resolved.

\section{Acknowledgements}

The authors wish to thank the OIF for its enthusiastic support for this project, and in particular, the leadership of Ms. Tracy S. Hart, CEO. We gratefully acknowledge the contributions of Angela M. Mancuso, Elizabeth J.

Simmonds, and Mr. Kyle Mulroy for their role in initiating this project and recruiting participation from the $\mathrm{Ol}$ community. We thank Winslow $\mathrm{M}$. Blankenship and Lauren Greco for their assistance in survey development, Cristine Agresta for her assistance with preliminary data analysis, and Cara Goerlich for her editing support. Beyond all measure, we are grateful and indebted to the adult $\mathrm{Ol}$ community for their personal generosity and patience without which this project would never have succeeded.

\section{Funding sources}

This project was supported by the Ernst and Gertrude Ticho Charitable Foundation, the George Washington University Medical Center Facilitating Fund, the Marshfield Clinic Center for Bone Disease, and Award Number UL1TR000075 from the NIH National Center for Advancing Translational Sciences. Its contents are solely the responsibility of the authors and do not necessarily represent the official views of the National Center for Advancing Translational Sciences or the National Institutes of Health.

\section{Author details}

'Division of Orthopedics and Sports Medicine, Children's National Health System, 111 Michigan Ave NW, Washington, DC 20010, USA. ²Osteogenesis Imperfecta Foundation, Gaithersburg, MD, USA. ${ }^{3}$ Rehabilitation Institute of Chicago, Chicago, IL, USA. ${ }^{4}$ Morris Animal Foundation, Denver, CO, USA. ${ }^{5}$ Temple University, Philadelphia, PA, USA. ${ }^{6}$ Marshfield Clinic, Chippewa Falls, WI, USA.

Received: 14 March 2015 Accepted: 28 October 2015

Published online: 14 November 2015

\section{References}

1. Inglefinger JR, Drazen JM. Patient organizations and research on rare diseases. N Engl J Med. 2011;364:1670-1.

2. Forlino A, Cabral WA, Barnes AM, Marini JC. New perspectives on osteogenesis imperfecta. Nat Rev Endocrinol. 2012;7:540-57.

3. Marini JC, Blissett AR. New genes in bone development: what's new in osteogenesis imperfecta. J Clin Endocrinol Metab. 2013;98(8):3095-103.

4. Byers PH, Pyott SM. Recessively inherited forms of osteogenesis imperfecta. Annu Rev Genet. 2012;46:475-97.

5. Fahiminiya S, Majewski J, Mort J, Moffatt P, Glorieux FH, Rauch F. Mutations in WNT1 are a cause of osteogenesis imperfecta. J Med Genet. 2013;50:345-8.

6. Retrouvey JM, Schwartz S, Hartsfield JK. Oral-facial aspects of osteogenesis imperfecta. In: Shapiro JR, Byers PH, Glorieux FH, Sponseller PD, editors. Osteogenesis imperfecta. A translational approach to brittle bone disease. London: Elsevier (Academic Press); 2014. p. 313-28.

7. McKiernan FE. Musculoskeletal manifestations of mild osteogenesis imperfecta in the adult. Osteoporos Int. 2005;16:1698-702.

8. Balkefors V, Mattsson E, Pernow Y, Saaf M. Functioning and quality of life in adults with mild-to-moderate osteogenesis imperfecta. Physiother Res Int. 2013;18:203-11.

9. Pillion JP, Shapiro J. Audiological findings in osteogenesis imperfecta. J Am Acad Audiol. 2008;19:595-601.

10. Chau FY, Wallace D, Vajaranant $T$, Herndon L, Lee $P$, Challa $P$, et al Osteogenesis imperfecta and the eye. In: Shapiro JR, Byers PH, Glorieux FH, Sponseller PD, editors. Osteogenesis imperfecta. A translational approach to brittle bone disease. London: Elsevier (Academic Press); 2014. p. 289-303.

11. Najib MQ, Schaff HV, Ganji J, Lee HR, Click RL, Miller DC, et al. Valvular heart disease in patients with osteogenesis imperfecta. J Card Surg. 2013;28:139-43.

12. Singer RB, Ogston SA, Patterson CR. Mortality in various types of osteogenesis imperfecta. J Insurance Med. 2001;33:216-20.

13. Shapiro J, Germain-Lee EL. Osteogenesis imperfecta: effecting the transition from adolescent to adult medical care. J Musculoskelet Neuronal Interact. 2012;12:24-7.

14. Zach P, DeVile C, Clark C, Surtees R. Understanding the information needs of general practitioners managing a rare genetic disorder (osteogenesis imperfecta). Community Genet. 2006;9:260-7.

15. Jones $L$, Wells K. Strategies for academic and clinician engagement in community-participatory partnered research. J Am Med Assoc. 2007;297:407-10 
16. Patient-Reported Outcomes Measurement System (PROMIS) roadmap initiative, National Institutes of Health. www.nihpromis.org. Accessed November 2015

17. Amor IMB, Roughly P, Glorieux FH, Rauch F. Skeletal clinical characteristics of osteogenesis imperfecta caused by haploinsufficiency mutations of COL1A1. J Bone Min Res. 2013;28:2001-7.

18. Liu HH, Cella D, Gershon R, Shen J, Morales LS, Riley W, et al. Representativeness of the PROMIS Internet panel. J Clin Epidemiol. 2010;63:1169-78.

19. Hays RD, Spritzer KL, Fries JF, Krishnan E. Responsiveness and minimally important difference for the Patient-Reported Outcomes Measurement Information System (PROMIS) 20-item physical functioning short form in a prospective observational study of rheumatoid arthritis. Ann Rheum Dis. 2015;74(1):104-7.

20. Yost KJ, Eton DT, Garcia SF, Cella D. Minimally important differences were estimated for six PROMIS-Cancer scales in advanced-stage cancer patients. J Clin Epidemiol. 2011;64:507-16.

21. http://www.cdc.gov/healthyweight/assessing/bmi/adult_bmi/. Accessed November 2015

22. Vital Health Stat 11. 2009 Apr;(249):1-68. http://www.cdc.gov/nchs/data/ series/sr_11/sr11_249.pdf. Accessed November 2014.

23. National Center for Health Statistics. Health, United States, 2011: With special feature on socioeconomic status and health. Library of Congress Number 76-641496. http://www.cdc.gov/nchs/data/hus/hus11.pdf. Accessed November 2015

24. The Eye Diseases Prevalence Research Group. Causes and prevalence of visual impairment in the United States. Arch Ophthalmol. 2004;122:477-85.

25. Go AS, Mozaffarian D, Roger VL, Benjamin EJ, Berry JD, Blaha MJ. American Heart Association Statistics Committee and Stroke Statistics Subcommittee. Heart disease and stroke statistics-2014 update: a report from the American Heart Association. Circulation. 2014;129(3):e28-292.

26. Siegel R, Naishadham D, Jemal A. Cancer statistics 2013. CA Cancer J Clin. 2013;63:11-30.

27. Higgins PDR, Johanson JF. Epidemiology of constipation in North America: a systematic review. Am J Gastroenerol. 2004;99:750-9.

28. Johanson JF, Sonnenberg A. The prevalence of hemorrhoids and chronic constipation; An epidemiologic study. Gastroenterol. 1990;98(2):380-6.

29. Chunh KF, Pavord ID. Prevalence, pathogenesis, and causes of cough. Lancet. 2008;371:1364-74.

30. Misery L, Sibaud V, Meriel-Kieny C, Taieb C. Sensitive skin in the American population; prevalence, clinical data, and the role of the dermatologist. Int J Derm. 2011:50:961-7.

31. Health United States 2005: with Chartbook on trends in the Health of Americans. National Center for Health Statistics (US), Hyattsville (MD) 2005.

32. Centers for Disease Control and Prevention. Percentage of adults aged 1864 years who have had problems with their teeth by race/ethnicity and type of problem. National Health Interview Survery, United States 2008. June 17, 2011/60(23)788

33. Camilleri M, Dubois D, Coulie B, Jones M, Kahrilas PJ, Rentz AM, et al. Prevalence and Socioeconomic impact of upper gastrointestinal disorders in the United States: Results of the US Upper Gastrointestinal study. Clin Gastro Hepatol. 2005;3:543-52.

34. Centers for Disease Control and Prevention. Self reported hypertension and use of antihypertensive medication among adults - United States, 2005-2009. Morb Mortal Wkly Rep. 2013;62:237-44

35. Scales CD, Smith AC, Hanley JM, Saigal CS. Prevalence of kidney stones in the Unites States. Eur Urol. 2012;14:154-7.

36. Mold JW, Vesely SK, Keyl BA, Schenk JB, Roberts M. The prevalence, predictors and consequences of peripheral sensory neuropathy in older patients. J Am Board Fam Pract. 2004;17:309-18.

37. Barbee RA, Halonen M, Kaltenborn WT, Burroughs B. A longitudina study of respiratory symptoms in a community sample population. Correlations with smoking, allergen skin-test reactivity, and serum IgE. Chest. 1991;99:20-6.

38. Lurie A. Obstructive sleep apnea in adults: epidemiology, clinical presentation and treatment options. Cardiol. 2011;46:1-42.

39. Patel RM, Nagamani SCS, Cuthbertson D, Campeau PM, Krischer JP, Shapiro $J R$, et al. A cross-sectional multi-center study of osteogenesis imperfecta in north america-results from the linked clinical research centers. Clin Gen. 2015:87(2):133-40.
40. Osteogenesis imperfecta: A guide for medical professionals, individuals, and families affected by OI. http://www.oif.org/site/DocServer/med_guide.pdf? docID=4501. Published Dec 2006. Accessed July 14, 2015

41. MCAllion SJ, Paterson CR. Causes of death in osteogenesis imperfecta. J Clin Pathol. 1996;49(8):627-30.

42. Engelbert RH, Gulmans VA, Uiterwaal CS, Helders PJ. Osteogenesis imperfecta in childhood: perceived competence in relation to impairment and disability. Arch Phys Med Rehabil. 2001;82(7):943-8.

43. Van Brussel M, Takken T, Uiterwaal CSPM, Prujis HJ, Van der Net J, Helders PJM, et al. Physical training in children with osteogenesis imperfecta. J Pediatr. 2008;152(1):111-6.

44. Sandhaus RA. Pulmonary function in osteogenesis imperfecta. In: Shapiro J, Byers PH, Glorieux FH, Sponsellor PD, editors. Osteogenesis imperfecta: a translational approach to brittle bone disease. London: Academic Press/ Elsevier; 2013. p. 335-42.

45. Thiele F, Cohrs CM, Flor A, Lisse TS, Przemeck GK, Horsch M, et al. Cardiopulmonary dysfunction in the Osteogenesis imperfecta mouse model Aga2 and human patients are caused by bone-independent mechanisms. Hum Mol Genet. 2012;21:3535-45.

46. Widmann RF, Laplaza FJ, Bitan FD, Brooks CE, Root L. Quality of life in osteogenesis imperfecta. Internat Orthop. 2002;26:3-6.

47. Bruce B, Fries J, Lingala B, Hussain YN, Krishnan E. Development and assessment of floor and ceiling items for the PROMIS physical function item bank. Arthritis Res Ther. 2013;15:R144.

48. Gosling SD, Vazire S. Should we trust web-based studies? Am Psychol. 2004:59(2):93-104

49. Vallejo MA, Mañanes G, Comeche MI, Díaz MI. Comparison between administration via Internet and paper-and-pencil administration of two clinical instruments: SCL-90-R and GHQ-28. J Behav Ther. 2008;29:201-8.

50. Mackenzie H, Thavaneswaran A, Chandran V, Gladman DD. Patient-reported outcome in psoriatic arthritis: a comparison of web-based versus papercompleted questionnaires. J Rheumatol. 2011;38(12):2619-24.

51. Fallows D. How women and men use the internet. Washington: The Pew Internet \& American Life Project; 2005. [2005 Dec 28]. http://www. pewinternet.org/files/2005/12/PIP Women_and Men_online.pdf.

52. Perrin A, Duggan M. Americans' internet access: 2000-2015. Washington: The Pew Internet \& American Life Project; 2015. [2015 June 26]. http://www. pewinternet.org/2015/06/26/americans-internet-access-2000-2015/.

53. Van Dijk FS, Sillence DO. Osteogeneis imperfecta: clinical diagnosis, nomenclature and severity assessment. Am J of Med Genet A. 2014;164A(6):1470-81.

54. Barton JL, Imboden J, Graf J, Glidden D, Yelin EH, Schillinger D. Patient-physician discordance in assessments of global disease severity in rheumatoid arthritis. Arth Care Res. 2010;62(6):857-64.

55. Markenson JA, Koenig AS, Feng JY, Chaudhari S, Zack DJ, Collier D, et al. Comparison of physician and patient global assessments over time in patients with rheumatoid arthritis: a retrospective analysis from the RADIUS cohort. J of Clin Rheumatol. 2013;19(6):317-23.

\section{Submit your next manuscript to BioMed Central and take full advantage of:}

- Convenient online submission

- Thorough peer review

- No space constraints or color figure charges

- Immediate publication on acceptance

- Inclusion in PubMed, CAS, Scopus and Google Scholar

- Research which is freely available for redistribution 